\title{
PESQUISA DE Cryptococcus neoformans EM INSTITUIÇÃO DE ENSINO SUPERIOR DA CIDADE DE CERES-GOIÁS
}

\section{Cryptococcus neoformans SEARCH IN THE INSTITUTION OF HIGHER EDUCATION OF CERES CITY -GOIÁS}

\author{
${ }^{1}$ Ana Clara dos Santos; ${ }^{1}$ Thays Ramos Silva; ${ }^{2,3}$ Renata Silva do Prado
}

\section{RESUMO}

Cryptococcus neoformansé um fungo basidiomiceto que se apresenta na forma tecidual como levedura capsulada, agente etiológico da Criptococose, uma micose humana sistêmica, podendo ser adquirida por via inalatória. Sabe-se que o fungo foi isolado e identificado em diferentes locais do Brasil, o que levanta preocupação sobre sua distribuição. Diante dessas informações e do fato de não existir nenhum estudo sobre a ocorrência de $C$. neoformans na cidade de Ceres - GO, o presente trabalho adquire relevância, sendo que se objetivou avaliar a presença de $C$. neoformans em excretas secas de pombos coletadas em uma instituição de ensino superior da cidade de Ceres-GO. Foi realizado um estudo de caráter qualitativo, de abordagem indutiva, com procedimento comparativo e técnica de documentação direta em laboratório. Foi realizada coleta de excretas secas de pombos no solo e bebedouro da Faculdade de Ceres (FACER) Unidade Ceres, posteriormente foi realizado preparo dessas amostras, e alíquotas desse preparo foram distribuídas em meio ágar Sabouraud Dextrose, onde pôde-se observar crescimento de microbiota fúngica, bem como teste da uréase, fenoloxidase e tinta nanquim. As análises das amostras coletadas, tanto em bebedouro como solo, apresentaram resultado negativo para a presença de Cryptococcus neoformans, em todas as metodologias utilizadas, confirmando sua ausência nos pombos que habitam o entorno do local de estudo.

Palavras-chave: Cryptococusneoformans. Criptococose. Excretas. Pombos.

\footnotetext{
${ }^{1}$ Acadêmica do curso de Farmácia, FACER- Faculdades de Ceres-GO, Brasil.

${ }^{2}$ Doutora em Medicina Tropical. Docente da FACER- Faculdades de Ceres-GO, Brasil. Docente da FACER Faculdades Unidade de Ceres-GO.

${ }^{3}$ Autora correspondente: Av. Brasil. SN, Qd. 13 Morada verde Ceres-GO CEP. 76300-000.

Fone- (62) 3323-1040. renata.ufg@ hotmail.com

Data do recebimento: 10/02/2016

Data da aprovação: 02/04/2016
} 


\begin{abstract}
Cryptococcus neoformansis a basidiomycete fungi that is presented in the form tissue as encapsulated yeast, etiological agent of cryptococcosis, systemic human mycosis and can be acquired by inhalation. It is known that the fungus was isolated and identified in different parts of Brazil, which raises concerns about its distribution. The fact that there is no study on the occurrence of $C$. neoformans in Ceres city, this work becomes relevant, and aimed to evaluate the presence of $C$. neoformans in dry feces of pigeons collected ina institution of higher education of Ceres-GO. It conducted a qualitative study of inductive approach, with comparative procedure and direct documentation technique in the laboratory. It was held collection of dried pigeon excreta in soil and water cooler of the Faculdade de Ceres (FACER) - Unidade Ceres, after it was made preparation of these samples, aliquot of this preparation were distributed in agar Sabouraud Dextrose, where it was observed growth fungal microbiota, and the urease test, phenoloxidase and India ink. The analysis of the samples collected, both as solo and water cooler, tested was negative for the presence of $C$. neoformans in all methodologies used, confirming their absence in pigeons that inhabit the surrounding place of study.
\end{abstract}

Key words: Cryptococcus neoformans. Cryptococcosis. Excreta. Pigeons.

\title{
INTRODUÇÃO
}

O fungo Cryptococcus neoformans é telemorfo pertencente ao filo Basidiomycota. Trata-se de um basidiomiceto que se apresenta na forma tecidual como levedura capsulada possuindo duas variedades fúngicas, tendo quatro sorotipos: variedade gattii (Sorotipos B e C) e variedade neoformans (Sorotipo A e B) (GOMPERTZ et al, 2008).

É um fungo ubíquo, que vive saprobioticamente no meio ambiente. Em sua variedade gattii é encontrada junto a eucaliptos, a variedade neoformans é encontrada em solos contaminados por fezes de pombos. É o agente etiológico da criptococose (DARZÉ et al, 2000). Criptococose é uma micose sistêmica causada pelo fungo oportunista C. neoformans, que pode ser adquirida via inalatória, quando os basidiósporos (forma infectante) ou leveduras desidratadas são aerolizadas e inaladas (MINISTÉRIO DA SAÚDE, 2012).

A forma pulmonar da doença pode ser classificada como infecção primária, que pode ser aguda, subaguda ou crônica. Essa infecção é de distribuição cosmopolita, e na maioria dos casos acomete indivíduos imunocomprometidos, que são mais susceptíveis a aquisição da 
infecção, como aqueles que fazem uso prolongado de corticosteroides ou possuem doenças autoimunes, tumores sólidos, transplantados, diabetes mellitus, doenças hepáticas e renais, que sejam residentes em áreas urbanas cujo habitat esteja associado aos locais que tenham fezes de pombos. Antes da AIDS ser conhecida mundialmente, a criptococose era doença de ocorrência rara (KWON-CHUNG et al, 1982.; LIZARAZO et al, 2007.; BATISTA et al, 2011). O tratamento da criptococose é realizado com os fármacos antifúngicos como a anfotericina $\mathrm{B}$ e o fluconazol (GULLO et al, 2012).

Relacionado à idade, a literatura vem mostrando uma maior frequência da infecção por C. neoformans na faixa etária entre 30 e 60 anos (SAAG et al, 2000).Quanto à distribuição mundial, estudos relataram um surto de criptococose em Vancouver, no Canadá, o que demonstra que representantes do gênero não se encontram apenas em regiões sub-tropicais ou tropicais, mas também em áreas temperadas, demonstrando sua distribuição pelas mais diferentes regiões do globo terrestre (BYRNES et al, 2010; HAGEN et al, 2010).

Estudos demonstraram que após 3 dias em temperatura de 25 a $37^{\circ} \mathrm{C}$, em cultivos nos meios ágar Sabouraud glicose $2 \%$ e ágar extrato de malte, ao microscópio, as leveduras de $C$. neoformans apresentam-se ovaladas ou globosas medindo cerca de 3 a $8 \mu \mathrm{m}$ de diâmetro, apresentando brotamento único ou múltiplo, de colo estreito e envolvidas por cápsula de mucopolissacarídeo. Mostra-se, macroscopicamente, como colônia de cor branca a creme, tem textura múcoide, brilhante, margem lisa e inteira (KON et al, 2008).

O presente trabalho teve como objetivo avaliar a presença de $C$. neoformans em excretas secas de pombos coletadas na Faculdade de Ceres (FACER) Goiás, através do cultivo em meio Ágar Sabouraud Dextrose, seguido do teste da Urease, teste da Fenoloxidase e o teste confirmatório da Tinta Nanquim.

\section{METODOLOGIA}

Foi realizado um estudo de caráter qualitativo, de abordagem indutiva, com procedimento comparativo e técnica de documentação direta em laboratório. A pesquisa foi realizada no laboratório multiuso da FACER-Unidade Ceres-GO.

\section{Coletas de Amostras}

Foram coletadas amostras de excretas secas de pombos, em bebedouros e solo da FACER- unidade Ceres, situada na região do vale do São Patrício.

De acordo com Faria e colaboradores (2010), as amostras foram raspadas e analisadas. Em cada ponto de coleta, com auxílio de espátulas, mascaras e luvas, foram colhidos entre 5 e 
50 gramas de excretas, de acordo com o volume encontrado. Os materiais foram acondicionados em frascos plásticos padronizados, em três triplicatas biológicas e identificados quanto à data e o local da coleta, e mantidos em refrigeração por até 48 horas.

\section{Preparo da amostra}

De cada amostra foram pesados $0,5 \mathrm{~g}$ de excretas secas de pombos e diluídas em $10 \mathrm{ml}$ de solução estéril salina $(0,85 \%)$ contendo amoxicilina na concentração $(0,05 \mathrm{~g} / \mathrm{L})$. A solução foi submetida a agitação em vórtex por 5 minutos, em seguida foi mantida em repouso por mais 5 minutos, para a decantação do material de não interesse, posteriormente foi coletado o sobrenadante que foi submetido a diferentes testes (CASALI et al; 2003), com modificações.

\section{Cultivos em Ágar Sabouraud Dextrose}

As amostras coletadas foram semeadas em meio Ágar Saboraud Dextrose (Peptona 10g/L; Dextrose 40g/L; Ágar 15g/L), meio recomendado para o cultivo de leveduras e fungos patogênicos (LACAZ et al., 1959; PACHECO et al, 2015). Para avaliação do possível crescimento de fungos e posterior identificação do gênero Cryptococcus.

\section{Teste da Uréase}

Seguindo a metodologia de Belli e colaboradores (2003), as amostras coletadas foram submetidas ao teste da uréase (Triptona 1,0g/L; Uréia 20,0g/L). As amostras colocadas em tubos separados com o meio da urease foram incubados a $37^{\circ} \mathrm{C}$ em banho maria por 24 horas, sendo considerados positivos os tubos onde existir viragem do meio de amarelo para vermelho, nas primeiras 6 horas de incubação.

\section{Teste da Fenoloxidase}

O ensaio para análise da enzima fenoloxidase, o qual identifica o gênero Cryptococcus, foi realizado a partir da adição de $100 \mu \mathrm{L}$ do sobrenadante da suspensão das fezes em solução salina na placa de Petri contendo Ágar Níger. As placas foram mantidas em estufa à $30^{\circ} \mathrm{C}$ e examinadas após 7 dias. Depois do crescimento das colônias, foram selecionadas as que apresentarem coloração marrom, já que esta cor é característica de colônias de Cryptococcus em Ágar Níger (GULLO, 2012). 


\section{Teste da Tinta Nanquim}

Conforme Silva (2004) e Gullo (2012), os fungos crescidos em Ágar Sabouraud Dextrose foram examinados ao microscópio óptico, após coloração com tinta da china, para identificação da cápsula polissacarídica e assim analisar o gênero encontrado.

\section{RESULTADOS E DISCUSSÃO}

A busca pelas potenciais fontes de contaminação por $C$. neoformans levou a um número grande de possibilidades, estando essas relacionadas a alguns substratos orgânicos, entre eles excretas de aves e vegetais. Assim, experimentos com meios de cultura ricos em substratos orgânicos favorecem seu crescimento, como o meio Ágar Sabouraud Dextrose (figura 1).

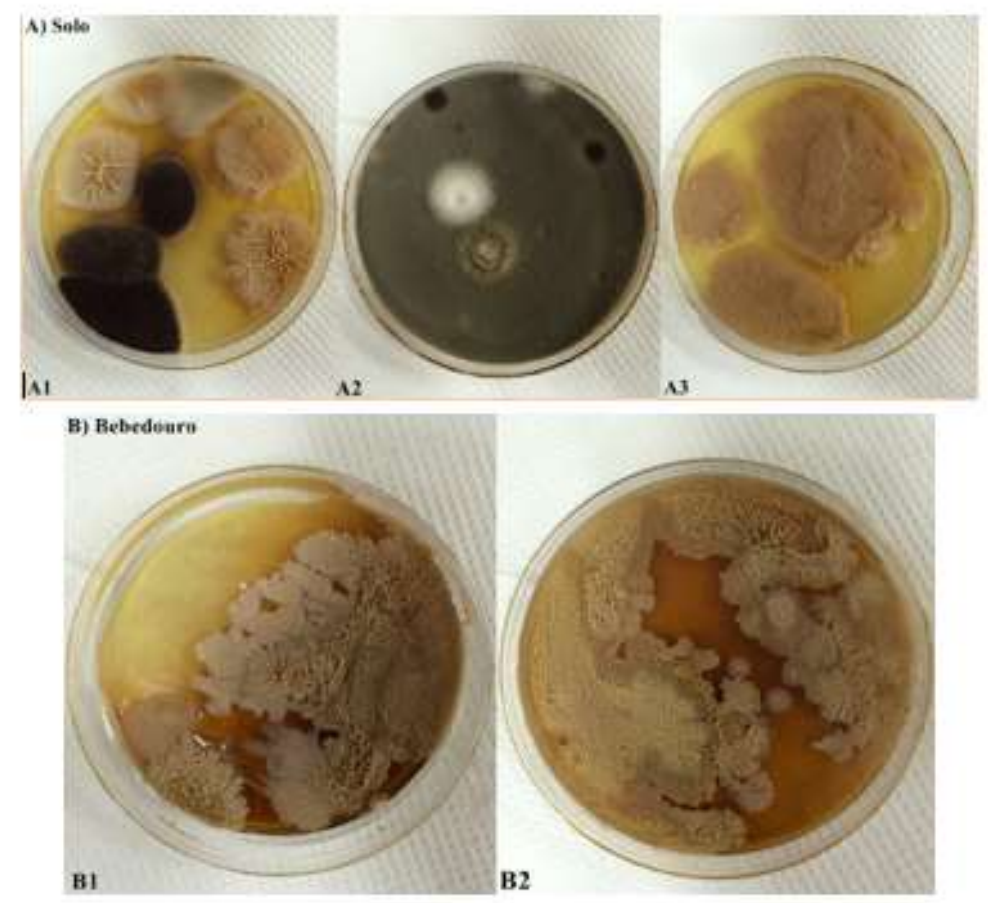

Figura 1: Cultivos de amostras de fezes de pombos em Ágar Sabouraud Dextrose. Em A) triplicata das amostras coletadas do solo em B)duplicata das amostras coletadas do bebedouro. Fotografadas após 7 dias de crescimento em estufa a $37^{\circ} \mathrm{C}$.

Como pode-se observar na figura 1 , foram encontrados vários tipos de colônias, algumas de textura cremosa, úmidas, com aspecto cerebriforme e cor creme, outras colônias boloriformes de coloração escura. Nota-se então, a presença dos dois tipos de colônias crescidas na mesma placa.

De acordo com o estudo de Baroni e colaboradores (2006), que avaliou a presença de C. neoformans em torres de igrejas na cidade do Rio de Janeiro (RJ), onde se tem registro da ocorrência de casos humanos dessa micose, observou-se em cultura a presença de colônias lisas, 
úmidas, brilhantes, de coloração creme a marrom e sem a presença de colônias emboloradas, que indica a presença de $C$. neoformans. Características determinantes como as colônias brilhosas e mucosas encontradas no estudo acima, não foram observadas neste trabalho.

Sabe-se que entre os fatores de virulência associados $C$. neoformans, pode-se citar a presença da enzima fenoloxidase, e da enzima uréase, responsável pela hidrólise da ureia (CASALI et al; 2003), ambas detectáveis através dos testes da uréase (figura 2) e fenoloxidase (dados não mostrados).

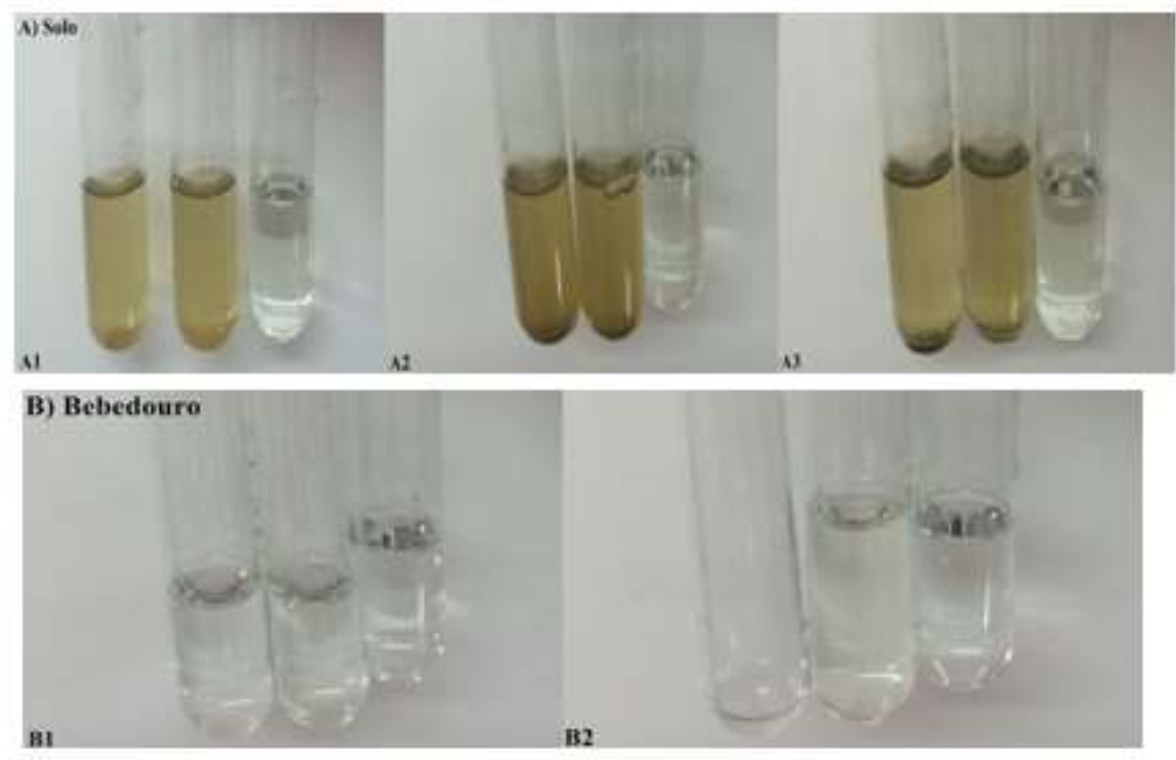

Fig2: Teste da Urease em Amostras de Fezes de Pombos. Em A) triplicatas das amostras coletadas do solo em B) triplicatas das amostras coletadas do bebedouro. Amostras incubadas em meio da uréase, a $37^{\circ} \mathrm{C}$ em banho maria por $24 \mathrm{~h}$, quando foram fotografadas.

Pode-se observar a partir da figura 2, o teste da uréase. Onde os tubos contendo meio de cultura mantiveram sua coloração marrom claro e transparente, depois de 24 horas a $37^{\circ} \mathrm{C}$, tanto para amostras de bebedouro quanto de solo, que permaneceram sem alterações na coloração, demonstrando ausência de atividade da enzima uréase.

Segundo Contin e colaboradoes (2010) e Mezzari e colaboradores (2014), que realizaram pesquisas na busca por $C$. neoformans, se faz necessária a realização de uma prova confirmatória de identificação, feita através do teste da uréase. Para que o teste seja positivo e necessário que haja uma viragem de cor como foi observado no trabalho de Contin, da coloração branca para cor rosa ou vermelho no final. Como não foi observado nenhuma viragem de cor no presente estudo, pode-se concluir que o teste teve resultado negativo, indicando que não há presença de $C$. neoformans nas amostras analisadas. 
O teste que detecta a ação da enzima fenoloxidase, é realizado em meio ágar Níger, e também foi negativo para presença de $C$. neoformans em todas as amostras analisadas (dados não mostrados).

As imagens da figura 3, demonstram o exame direto da tinta da china ou tinta nanquim. Foi feito uma varredura por toda a lâmina e não se pôde observar a presença de cápsulas. Devido a coloração da tinta a figura apresentou fundo preto e ao centro podemos notar a presença de algumas bolhas com a ausência central do fungo.
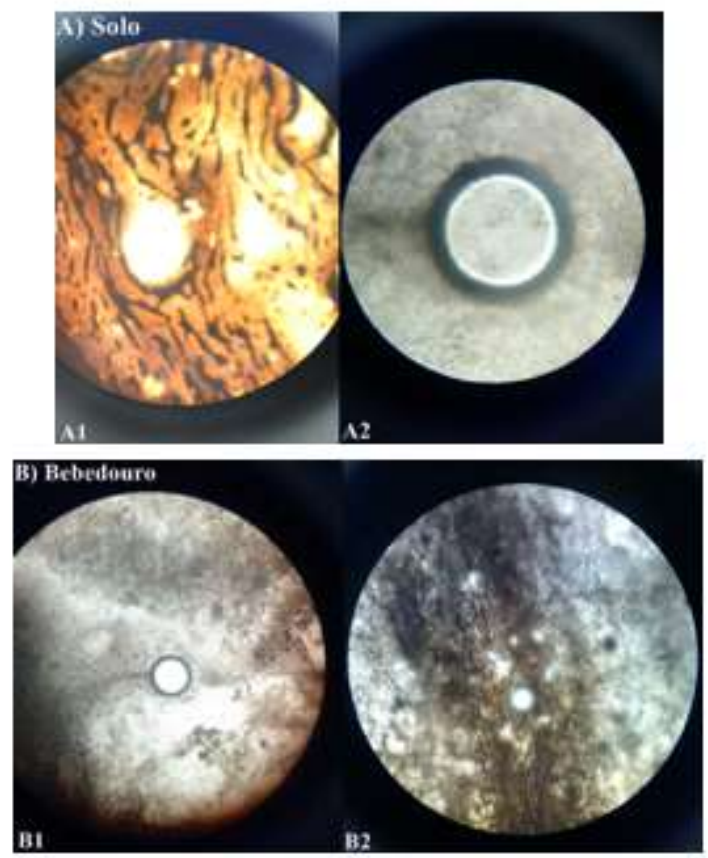

Fig3: Teste da Tinta da China em Amostras de Fezes de Pombos. Em A) duplicata das amostras coletadas em solo em B)duplicata das amostras coletadas no bebedouro. Os fungos crescidos em ágar sabouraud dextrose foram levados ao microscópio e observados com aumento de 400x.

Segundo Faria e colaboradores (2010) e Filiu e colaboradores (2002) que realizaram pesquisa por $C$. neoformans para que o exame direto de tinta da china ou nanquim seja positivo é necessário encontrar células redondas ou ovais, envolvidas por uma cápsula de espessura variável, com ou sem brotamento, unibrotantes ou multibrotantes e sem hifas ou pseudo-hifas, como não há presença de um núcleo nas imagens obtidas, constata-se que não há presença de C. neoformans nas excretas analisadas.

Resultado diferente foi obtido por Araújo-Júnior (2014), que ao fazer o levantamento em locais de possível risco da cidade de Araçatuba, SP, encontrou resultados positivos tanto 
para $C$. neoformans quanto para $C$. gatti, levantando a necessidade de um programa de conscientização e novas normas de higiene e limpeza para os locais analisados.

\section{CONCLUSÃO}

Os resultados obtidos neste estudo permitem verificar a ausência de C. neoformans nos pombos que habitam o entorno da FACER-Ceres, uma vez que não foram encontrados propágulos em nenhuma das amostras analisadas (solo e bebedouro).

Estes achados trazem tranquilidade no que se refere as condições higiênico-sanitárias das excretas no local de análise. No entanto, estudos que monitorem a existência de $C$. neoformans em locais de risco, como as praças públicas da cidade, ainda se fazem necessários.

\section{REFERÊNCIAS}

BARONI, F.A.; PAULA, C.R.; SILVA, E.G; VIANI, F.C; RIVERA I.N.G; OLIVEIRA, M.T.B.; GAMBALE,W.CryptococcusNeoformansem torres de igreja da cidade do Rio de Janeiro, RJ, Brasil. Rev. Inst. Med. Trop. S. Paulo, v.48, n.2, abril 2006.

BATISTA, M.V.; PIERROTTI, L.C.; ABDALA, E.; CLEMENTE, W.T.; GIRÃO, E.S.; ROSA, D.R.; IANHEZ, L.E.; BONAZZI, P.R.; LIMA, A.S.; FERNANDES, P.F.; PÁDUANETO, M.V.; BOCCHELLA, T.; OLIVEIRA, A.P.; VIANA, C.F.; FERREIRA, M.S.; SHIKANAI-YASUDA, M.A. Endemicandopportunisticinfections in Braziliansolidorgantransplantrecipients. TropMedInt Health, v. 16, n. 9, p. 1134-1142, 2011.

BELLI, C. B.; FERNANDES, W. R.; SILVA, L. C. L. C. Teste da uréase positivo em equinos adultos com ulcera gástrica - Helicobacter SP. Arq. Inst. Biol., v.70, n.1, p.17-20, 2003.

BYRNES, E.J.; LI, W.; LEWIT, Y.; MA, H.; VOELZ, K.; REN, P.; CARTER, D.A.; CHATURVEDI, V.; BILDFELL, R.J.; BILDFELL, R.J.; MAY, R.C.; HEITMAN, J. Emergence and pathogenicity of highly virulent Cryptococcus gattii genotypes in the northwest United States. PLoSPathog, v. 6, p. 1000850 - 40, 2010.

CASALI, A. K.; GOULART, L.; SILVA, L.K.R.; RIBEIRO, A.M.; AMARAL, A.A.; ALVES, S.H.; SCHRANK, A.; MEYER, W.; VAINSTEIN, M.H. Molecular typing of clinical and environmental Cryptococcus neoformans isolates in the Brazilian state Rio Grande do Sul. FEMS Yeast Res, v. 3, n. 4, p. 405-415, 2003.

CONTIN, J.T.; QUARESMA, G.S.; SILVA, E. F.; LINARDI, V.R. Ocorrência de cryptococcusneoformans em fezes de pombos na cidade de Caratinga, MG - Brasil. RMMG (Revista medica de Minas Gerais), v.21, 2010. 
DARZÉ, C.; LUCENA, R.; GOMES, I.; MELO, A. Características clínicas laboratoriais de 104 casos de meningoencefalitecriptocócica. Revista da Sociedade Brasileira de Medicina Tropical, v. 1, n. 33, p. 21-26, 2000.

FARIA, R. O.; NASCENTE, P. S.; MEINERZ, A. R. M.; CLEFF, M. B.; ANTUNES, T. A.; SILVEIRA, E. S.; NOBRE, M. O.; MEIRELES, M. C. A.; MELLO, J. R. B.Ocorrência de Cryptococcusneoformans em excretas de pombos na cidade de Pelotas, Estado do Rio Grande do Sul. Revista da Sociedade Brasileira de Medicina Tropical, v. 43, n. 2, p. 198$200,2010$.

FILIÚ, W.F.O.; WANKE, B.; AGUENA, S.M; VILELA, V.O; MACEDO, R.C.L.; LAZERA, M. Cativeiro de aves como fonte de CryptococcusNeoformans na cidade de Campo Grande, Mato Grosso, Brasil. Revista da Sociedade Brasileira de Medicina Tropical, v.35, p.591595, Nov-dez, 2002.

GOMPERTZ,O.F.;GAMBALE,W.;PAULA,C.R.;CORRÊA,B. Micoses Sistêmicas. In: TRABULSI,L.R.; ALTERTHUM,F. Microbiologia. Editora Atheneu, 2008.

GULLO, F.P. Antifungicos Naturais e Sinteticos: Estudo dos mecanismos de ação em sistema de infecção IN VITRO, empregando cepas de Cryptococcus.2012, 140 f. Dissertação (Mestrado em Biociências e Biotecnologias Aplicadas a Farmácia) - Faculdade de Ciências Farmacêuticas, Universidade Estadual Paulista - UNESP, Araraquara, São Paulo.

HAGEN, F.; ILLNAIT-ZARAGOZI, M.T.; BARTLETT, K.H.; SWINNE, D.; GEERTSEN, E.; KLAASSEN, C.H.W.; BOEKHOUT, T.; MEIS, J.F. In Vitro Antifungal Susceptibilities and Amplified Fragment Length Polymorphism Genotyping of a Worldwide Collection of 350 Clinical, Veterinary, and Environmental Cryptococcus gattii Isolates. AntimicrobAgentsChemother, v.54, p. 5139- 5145, 2010.

JUNIOR, E.C.A. Cryptococcus: Isolamento Ambiental e Caracterização Bioquímica. 2014, 52 f. Dissertação (Mestrado em Ciência Animal) - Faculdade de Medicina Veterinária, Universidade Estadual Paulista - UNESP, Araçatuba, São Paulo.

KON, A. S.; GRUMACH, A. S.; COLOMBO, A. L.; PENALVA, A. C. O.; WANKE, B.; TELLES, F. A.; SEVERO, L. C.; ARANHA, L. F.; LAZÉRA, M. S.; RESENDE, M. R.; SALMITO, M. A.; YASUDA, M. A. S.; MOREHI, M. L.; FERREIRA, M. S.; VERGARA, M. L. S.; ANDRADE, N. M. P.; TRABASSO, P.; MENDES, R. P.; MARTINEZ, R.; PONZIO, V. Consenso em criptococose. Revista da Sociedade Brasileira de Medicina Tropical, v. 41, n. 5, p. 524-544, 2008. 
KWON-CHUNG, K. J.; POLACHECK, I.; BENNETT, J. E. Improved diagnostic medium for separation of Cryptococcusneoformans var. neoformans (serotypes A and D) and Cryptococcus neoformans var. gattii (serotypes B and C). J ClinMicrobiol, v. 15, n. 3, p. 535-537, 1982.

LACAZ, C. S.;ULSON, C. M.; SAMPAIO S. A. P. Ação in vitro da anfotericina B sobre o $P$. brasiliensis. Rev. Paul. Med., v.54,p. 357-360, 1959.

LIZARAZO, J.; LINARES, M.; BEDOUT, C.; RESTREPO, A.; AGUDELO, C.I.; CASTANEDA, E. Estudio clínico y epidemiológico de lacriptococosisenColombia: resultados de nueveaños de laencuesta nacional, 1997-2005. Biomedica, v. 27, n. 1, p. 94-109, 2007.

MEZZARI, A.; WLIEBBELLING, A.M.P.; WENCZENOVICZ, C.; SOUZA, C.D.A.; FREITAS, G.S.O.; BARBOSA, L.D.; PENA, L.D.; KISSMANN, N.; PORTICH, J.P.; CARNEIRO, L.C.; BEHAR, P.R.P. Presença do Cryptococcus spp. nas excretas de pombos nos arredores de Hospitais de Porto Alegre. Rev Panam Infectol, v.16, p:153-160, 2014.

MINISTÉRIO DA SAÚDE. Vigilância Epidemiológica da Criptococose. Ministério da Saúde, Brasília, 2012.

PACHECO, D.R.; SOARES, D.E.D.; NETO, C.M.S.; SILVA, G.A.; PRADO, R.S. Avaliação da atividade antifúngica de curcuna longa sobre cândida parapsilosis. Res Patol Trop, v.44, p.258-270, 2015.

SAAG, M.S.; GRAYBILL, R.J.; LARSEN, R.A.; PAPPAS, P.G.; PERFECT, J.R.; POWDERLY, W.G.; SOBEL, J.D.; DISMUKES, W.E. Practice guidelines for the management of cryptococcal meningitis. Clinical of Infectious Diseases, v. 30, p. 710-718, 2000.

SANTOS, L. L.; FERREIRA, F. M.; LOPES, S. F.; CONDAS, L. A.; MURO, M. D.; LUGARINI, C. Pesquisa de Cryptococcusneoformans e Candida spp. em excretas de psitacídeos e passeriformes cativos. Arq. Ciênc. Vet. Zool. Unipar, v. 12, n. 1, p. 5-9, 2009.

SILVA, R. M. G. Meningite por Criptococcusneoformans como causa de febre prolongada em paciente com AIDS. Revista da Associação Médica Brasileira, v. 50, n. 2, p. 109-26, 2004. 